Article

\title{
Outdoor Node Localization Using Random Neural Networks for Large-Scale Urban IoT LoRa Networks ${ }^{\dagger}$
}

\author{
Winfred Ingabire ${ }^{1,2}$, Hadi Larijani ${ }^{1, *} \mathbb{B}$, Ryan M. Gibson ${ }^{1}$ and Ayyaz-UI-Haq Qureshi ${ }^{1}$ \\ 1 School of Computing, Engineering and Built Environment, Glasgow Caledonian University, \\ Glasgow G4 0BA, UK; Winfred.Ingabire@gcu.ac.uk (W.I.); Ryan.Gibson@gcu.ac.uk (R.M.G.); \\ Ayyaz.qureshi@gcu.ac.uk (A.-U.-H.Q.) \\ 2 Department of Electrical and Electronics Engineering, College of Science and Technology, University of \\ Rwanda, Kigali P.O. Box 4285, Rwanda \\ * Correspondence: H.Larijani@gcu.ac.uk \\ + This paper is an extended version of our paper published in 2021 Computing Conference, London, UK, \\ 15-16 July 2021.
}

Citation: Ingabire, W.; Larijani, H.; Gibson, R.M.; Qureshi, A.-U.-H. Outdoor Node Localization Using Random Neural Networks for Large-Scale Urban IoT LoRa Networks. Algorithms 2021, 14, 307. https://doi.org/10.3390/a14110307

Academic Editors: Frank Werner and Apostolos Gkamas

Received: 30 August 2021

Accepted: 21 October 2021

Published: 23 October 2021

Publisher's Note: MDPI stays neutral with regard to jurisdictional claims in published maps and institutional affiliations.

Copyright: () 2021 by the authors. Licensee MDPI, Basel, Switzerland. This article is an open access article distributed under the terms and conditions of the Creative Commons Attribution (CC BY) license (https:// creativecommons.org/licenses/by/ $4.0 /)$.

\begin{abstract}
Accurate localization for wireless sensor end devices is critical, particularly for Internet of Things (IoT) location-based applications such as remote healthcare, where there is a need for quick response to emergency or maintenance services. Global Positioning Systems (GPS) are widely known for outdoor localization services; however, high-power consumption and hardware cost become a significant hindrance to dense wireless sensor networks in large-scale urban areas. Therefore, wireless technologies such as Long-Range Wide-Area Networks (LoRaWAN) are being investigated in different location-aware IoT applications due to having more advantages with low-cost, long-range, and low-power characteristics. Furthermore, various localization methods, including fingerprint localization techniques, are present in the literature but with different limitations. This study uses LoRaWAN Received Signal Strength Indicator (RSSI) values to predict the unknown X and Y position coordinates on a publicly available LoRaWAN dataset for Antwerp in Belgium using Random Neural Networks (RNN). The proposed localization system achieves an improved high-level accuracy for outdoor dense urban areas and outperforms the present conventional LoRa-based localization systems in other work, with a minimum mean localization error of $0.29 \mathrm{~m}$.
\end{abstract}

Keywords: IoT; LoRaWAN; RSSI; localization; RNN

\section{Introduction}

Localization is a vital research subject that is gaining popularity and being applied in different IoT location-based applications, such as tracking. Moreover, various wireless technologies are being applied in localization. WiFi [1], Bluetooth [2], and Zigbee [3] are primarily applied in localization systems for indoor environments and only limited to a few meters. Furthermore, technologies based on satellites are the pioneers in the accurate localization systems for outdoor applications, with errors less than $4 \mathrm{~m}$ for Galileo's Open Service and $10 \mathrm{~m}$ using GPS [4]. Satellite-based technologies provide continuous global coverage and play significant roles in a wide range of applications. However, Global Navigation Satellite System (GNSS) modules consume high energy, which rules them out for many low-power IoT end devices. Additionally, incorporating many stand-alone sensor nodes in dense sensor network applications with GPS modules would not be efficient due to high hardware cost, high power consumption, and failures due to non-line-of-sight communications [5]. Time-of-Arrival (ToA), RSSI ranging, and Time-Difference-of-Arrival (TDoA) algorithms are among the most researched range-based localization techniques in IoT and Wireless Sensor Networks (WSN). Trilateration or Triangulation approaches are used to calculate the location coordinates of the end device using multiple anchor end nodes with known location coordinates [6], whereby LoRaWAN gateways may be referred 
to as anchor points. A propagation model and the RSSI are used to compute the distance between receiver and transmitter in RSSI ranging. However, multipath and shadow fading lead to the loss profile with indoor RSSI ranging.

A scheduling algorithm with accurate end devices' time synchronization is required for ToA, whereas accurate gateways time stamps for at least three distant gateways are only required for TDoA and make it more suitable for IoT end devices. The calculation of an end device location is made when at least three distant gateways received the same transmission signal from the same end device as, ideally, the signal trajectories to the gateways intersect at the location of the end device. However, any slight scheduling error may lead to inaccurate location predictions. The traditional Low-power Long-Range (LoRa)-based localization systems use TDoA [7] and fingerprint algorithms [8], all with limitations in dense, large urban areas and still under investigation. TDoA performance is poor in dense urban areas, though it performs well in open areas [9]. Fingerprinting localization uses end devices' RSSI fingerprint values received by gateways to localize any end device in the network accurately. A fingerprint-based approach consists of an offline training phase and an online phase [10]. Data samples are collected from a considered service area where localization is to be predicted and used to train a fingerprint algorithm. The location of any end device can then be predicted using the trained algorithm in an online phase based on end-device RSSI characteristics. However, all existing fingerprint algorithms also have various challenges; among others are changing environments that affect fingerprint maps, fingerprint databases that require manpower to be created, and the complicated infrastructure layout, particularly in urban areas. Additionally, different RSSI fingerprint Low-Power Wide-Area Networks (LPWAN) such as LoRaWAN-based approaches using different machine learning algorithms and Artificial Neural Networks (ANN) are also present in the literature [11].

Furthermore, RNN has been used to develop robust models in different applications with considerable accuracy [12]. However, RNN applied to localization systems is a research topic yet to be fully explored. This research applies RNN to develop a lowpower, large-scale localization system using LoRaWAN RSSI values to predict unknown 2D X and Y coordinates. Different RNN-based localization models are trained and tested using different learning rates and samples on the publicly available LoRaWAN dataset for Antwerp in Belgium and outperforms localization systems in other related work with the minimum mean localization error of $0.29 \mathrm{~m}$. The main contributions of this paper are as follows:

- Developing a new LoRaWAN-based localization model using RNN for large, dense urban scenarios.

- Training and testing the different RNN-based localization systems with various learning rates.

- Training and testing the different RNN-based localization models with different data samples in Antwerp dataset publicly available and used in many research papers.

- Critically analyzing the results with other popular methods applied to the same dataset.

The organization of this paper is as follows: Section 2 gives an overview of LoRa and LoRaWAN; Section 3 summarizes the related work; Section 4 describes the methodology used; Section 5 discusses the obtained results and performance analysis; finally, conclusions are given in Section 6.

\section{LoRa and LoRaWAN}

LoRa is a physical layer with the Chirp Spread Spectrum (CSS) modulation technique operated by Semtech and usable within the license-free spectrum from $863 \mathrm{MHz}$ to $870 \mathrm{MHz}$ in Europe and from $902 \mathrm{MHz}$ to $928 \mathrm{MHz}$ in the USA [13]. The connection of the widearea network of LoRa is called LoRaWAN and is a network protocol stack and offers the architecture of LoRa technology on the MAC layer. A LoRaWAN network is made up of LoRa end devices connected in a star topology that sends information to one or more LoRaWAN gateways, which, in turn, send the received message along with a recorded 
unique message's metadata information to a network server, as shown in Figure 1. This metadata information is used for localization services in LoRaWAN networks, whereby gateways serve as anchor points to predict end device locations. LoRaWAN networks use RSSI values as a critical metric for fingerprint localization algorithms, whereas TDoA algorithms use timestamps at which gateways receive the same message. Furthermore, localization accuracy of any of the methods depends on more gateways receiving the same message [14].

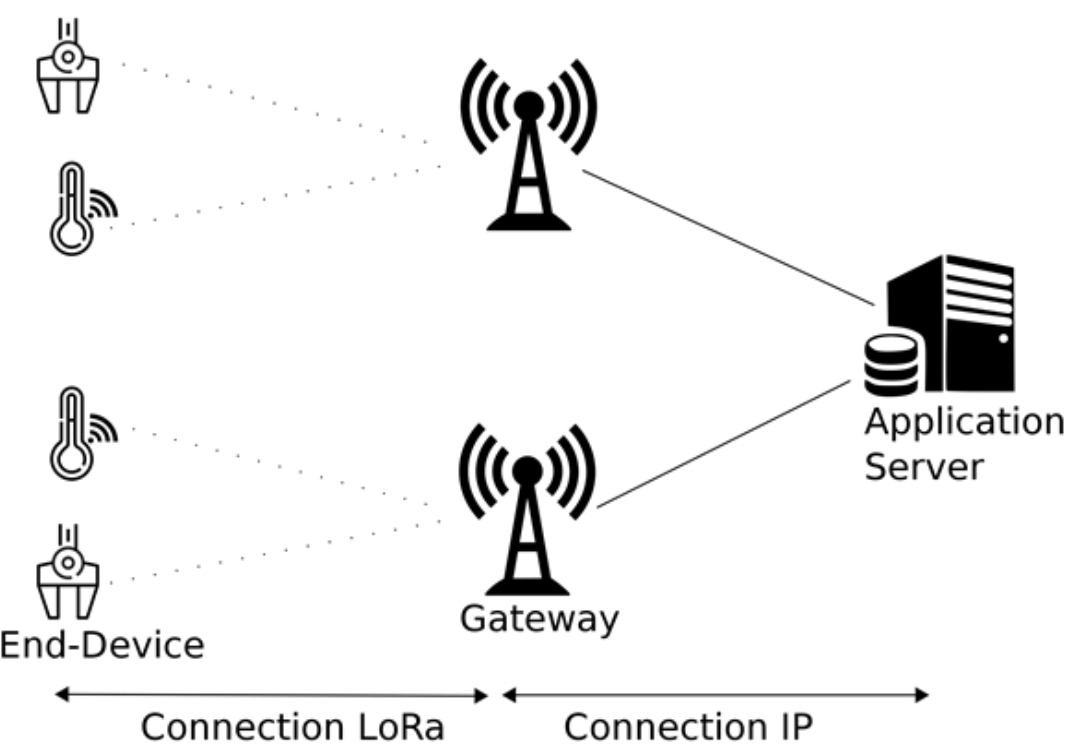

Figure 1. LoRaWAN architecture [13].

\section{Related Work}

Several TDoA-based localization systems using LoRaWAN IoT networks are available in the literature $[10,15]$. An acceptable accuracy of less than $100 \mathrm{~m}$ in most of the published work was reported considering fixed nodes and gateways as anchor points on a small area. However, the performance degraded significantly when mobile nodes or large areas were considered. Fargas et al. in [16], used an iterative TDoA-based algorithm to locate static nodes and obtained a good accuracy with an error of around $100 \mathrm{~m}$. A median error of $200 \mathrm{~m}$ was reported by Podevijn et al. in [7] by using a TDoA-based algorithm that considered map details such as roads. Aernouts. M. et al. in [17], via extensive simulations, used two gateways with combined TDoA and Angle of Arrival (AoA) using probabilistic algorithms. Their simulation results reported a mean error of $548 \mathrm{~m}$ with TDoA and managed to reduce the mean error to $399 \mathrm{~m}$ by combining the TDoA estimate with a single AoA estimate. Furthermore, range-based algorithms do not work in indoor scenarios due to multipath as a result of complicated radio environments. Hence, RSSI fingerprinting localization techniques also explored and proved to be potential candidates for harsh environments, including both indoor and outdoor dense urban areas. Different algorithms using RSSI fingerprint localization are available in the literature using various technologies; nevertheless, most of the present works investigated indoor scenarios because of many data needed for the training phase and tedious work in accumulating enough data for a large area. Wi-Fi has been used in fingerprint localization by different researchers [18-21], whereby a smartphone may be used to record its RSSI and calculate its location using the web. The authors in [22] compared the performance analyses of different wireless technologies based on RSSI localization. Furthermore, the authors in [23] used satellite images for LoRa-based outdoor fingerprint localization and achieved a median error of $47.1 \mathrm{~m}$.

Different research works in the literature have evaluated ANN methods for sensor localization and confirmed them to be effective [24]. In addition, the authors investi- 
gated localization models using ANN for Low-Power Wide Area Networks (LPWAN) in [25], and they confirmed ANN as an objective approach, mainly for dense IoT networks. Furthermore, various studies are available in the literature on using ANN to develop LoRa-based $[8,26,27]$ localization models with high accuracy. Different RSSI fingerprint LPWAN-based approaches using machine learning algorithms, particularly LoRaWAN, are also present in the literature [11]. Janssen. T. et al. in [17] conducted a comparative performance analysis of various machine learning algorithms for RSS-LPWAN-based localization models. The random forest regression method had the highest accuracy with a mean estimation error of $340 \mathrm{~m}$, and the k-Nearest Neighbour ( $\mathrm{kNN}$ ) method had a similar accuracy with the least computational performance. Sallouha reported an error lower than $20 \mathrm{~m}$ in [28], while analyzing localization in ultra-narrow band IoT networks. Similarly, RSSI fingerprint localization methods based on deep learning algorithms have also been published in the literature [29-32]. Moreover, Carrino. F. et al. in [33] reported a root mean square estimation of error less than $9 \mathrm{~m}$ with a Long Short-Term Memory (LSTM) method compared with the accuracy of Random Forest and ANN methods.

Furthermore, RNN algorithms have been used in various applications including Heating, Ventilation, and Air Conditioning (HVAC) systems [34-36]; nonoccupied buildings' energy prediction [37]; image pattern recognition [38]; and intrusion detection systems [12,39,40] all with significant results. Moreover, according to the results of Cerkez et al. [41] and Abdelbaki et al. [42], simple encounters can represent neurons in RNN and, hence, easy hardware implementation. In addition, RNN accurately predicted unseen patterns not included in the training data when compared with the conventional ANN performance [43]. RNN outperformed ANN during run-time though at the expense of a greater training time [44]; the authors also reported that $\mathrm{RNN}$ had a stronger generalization capacity for the training phase uncovered patterns. Furthermore, a performance analysis of different models of the proposed RNN-based localization system was evaluated in our previous published work [45]. Nevertheless, RNN application is still under investigation and yet to be fully applied in developing and evaluating end device localization systems in general, specifically using LoRaWAN in a large, dense urban environment.

\section{Methodology}

This section presents all the details and procedures used to collect and preprocess the dataset we used to develop the proposed LoRa-based localization system using RNN.

\subsection{Dataset}

Our study used the LoRaWAN dataset published by Aernouts et al. in [46], gathered in an area of $52 \mathrm{~km}^{2}$ in Antwerp in Belgium, and is publicly available. Data were collected by attaching LoRa modules to postal service vehicles, whereby a total of 130,343 data points were gathered from these nodes sending a message every minute to 72 LoRaWAN gateways deployed by a private company called Proximus for three months. A map showing a random sample of data points in Antwerp evenly distributed in the city streets is shown in Figure 2. For every data point or message, the authors recorded $X$ and $Y$ position coordinates of each LoRa node with its RSSI values received by 72 different gateways. If any of the gateways did not receive a particular message, a RSSI value of -200 was recorded. The distribution of RSSI values is presented in Figure 3 and varies between $-122 \mathrm{dBm}$ and $-79 \mathrm{dBm}$. 


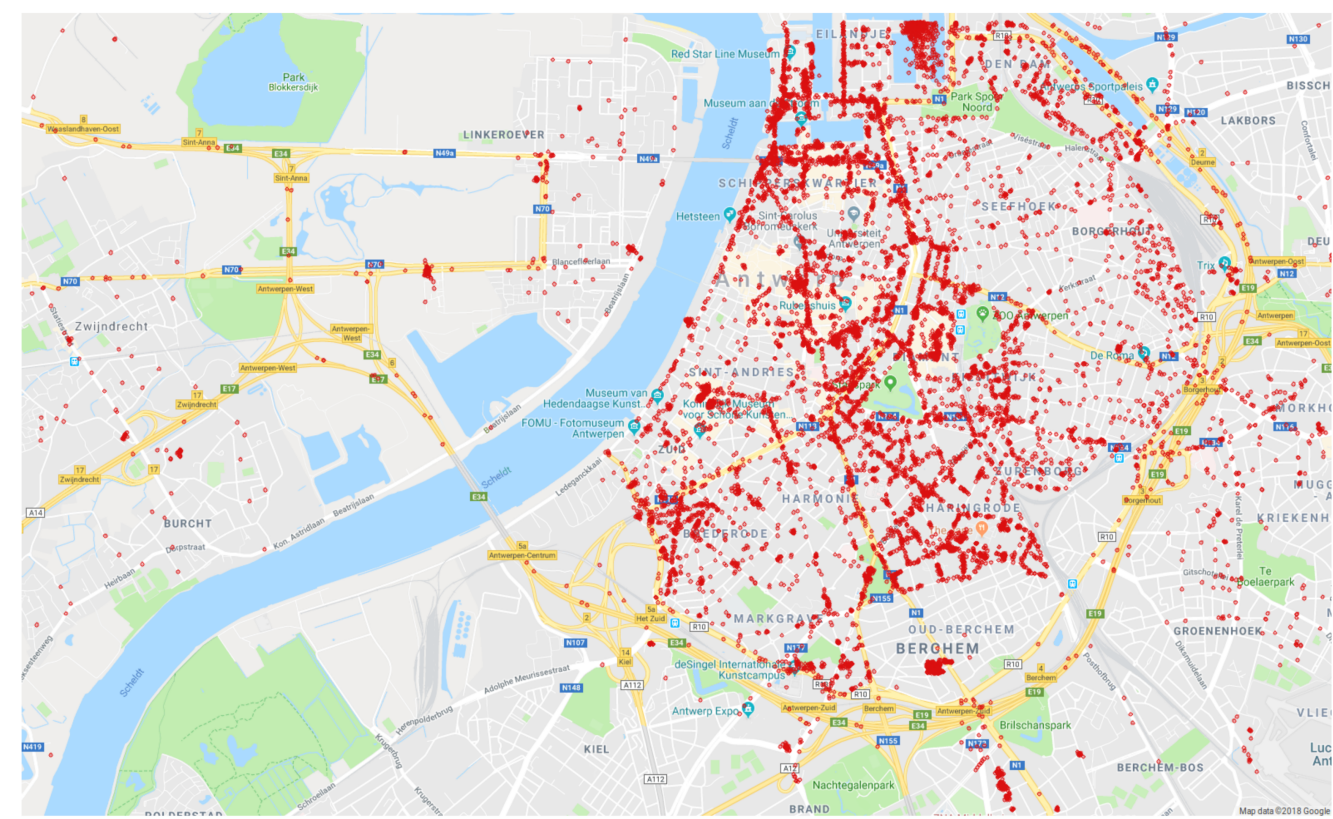

Figure 2. A map showing a random sample of used data points [46].

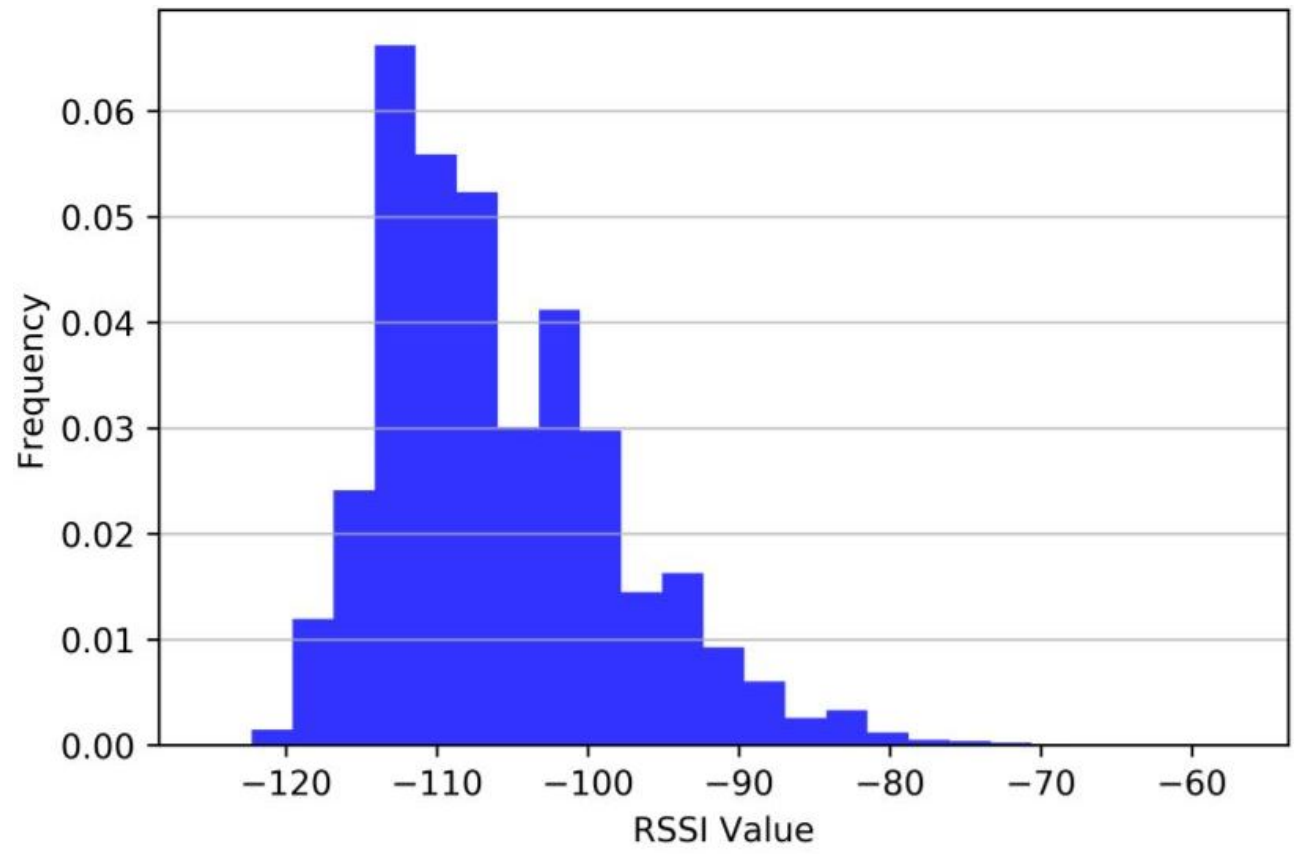

Figure 3. The distribution of RSSI Values for Antwerp.

\subsection{Data Normalization}

The RSSI values of the used dataset are large and, due to large weights, the network becomes unstable. Therefore, we scaled the dataset using the Min-Max Normalization data preprocessing technique to the range of 0 to 1 , with the formula that follows:

$$
x_{i}=\frac{R S S I_{i}-\min (R S S I)}{\max (R S S I)-\min (R S S I)},
$$

whereby the raw RSSI input data is $R S S I=\left(R S S I_{1}, \ldots, R S S I_{n}\right)$ and the resultant normalized data are $x(i)$. 


\subsection{Proposed RNN-Based Localization System Using LoRaWAN}

Gelenbe developed RNN as a novel class of ANN [47]. It is composed of N several layers of linked neurons that exchange information signals as impulses; a positive potential $(+1)$ is used for an excited signal and a negative potential $(-1)$ is used for inhibited signals to the next connected neuron. The potential of every neuron $i$ at time $t$ is represented by a nonnegative integer $K_{i}(t)$. The neuron $i$ is in an excited state if $K_{i}(t)>0$ and I is in idle state if $K_{i}(t)=0$. If neuron $i$ is excited, it transmits signal information to the next receiving neuron $j$ at the Poisson rate $r_{i}$. The transmitted signal can reach neuron $j$ as an impulse signal in an excited or inhibition state with probabilities $p^{+}(i, j)$ or $p^{-}(i, j)$, respectively. Furthermore, the transmitted signal can leave the network with a probability defined with the following mathematical formula:

$$
\begin{gathered}
c(i)+\sum_{j=1}^{N} p^{+}(i, j)+p^{-}(i, j)=1, \forall i, \\
w^{+}(i, j)=r_{i} p^{+}+(i, j) \geq 0,
\end{gathered}
$$

Likewise

$$
w^{-}(i, j)=r_{i} p^{-}+(i, j) \geq 0 .
$$

Equations (2)-(4) combined:

$$
r(i)=(1-c(i))^{-} 1 \sum_{j=1}^{N}\left[w^{+}(i, j)+w^{-}(i, j)\right]
$$

The rate of transmission between neurons in Equation (5) is $r(i)$, and is defined as $r(i)=\sum_{j=1}^{N}\left[w^{+}(i, j)+w^{-}(i, j)\right]$. Though " $w^{\prime \prime}$ describes the matrices of weight updates from neurons, it is always positive as it is a product of probabilities and transmission rates.

If a signal arrives at neuron $(i)$ in excitation state with a positive potential, it is denoted by Poisson rate $\Lambda(i)$, while a signal in inhibition with a negative potential reaches at a Poisson rate $\lambda(i)$. Hence, for each node " $i$ ", the output activation function for that particular neuron is described by

$$
q(i)=\frac{\lambda^{+}(i)}{r(i)+\lambda^{-}(i)}
$$

where

$$
\lambda^{+}(i)=\sum_{j=1}^{n} q(j) r(j) p^{+}(j, i)+\Lambda(i)
$$

and

$$
\lambda^{-}(i)=\sum_{j=1}^{n} q(j) r(j) p^{-}(j, i)+\lambda(i)
$$

In this study, the proposed RNN-LoRaWAN-based localization system is trained with Gradient Descent (GD) and the computed weights and biases are updated to the neurons as the algorithm calculates the error. GD is a first-order iterative optimization algorithm commonly considered by various researchers for training; it minimizes the cost function, and the error cost function is described by

$$
E_{p}=\frac{1}{2} \sum_{i=1}^{n} \gamma_{i}\left(q_{j}^{p}-q_{j}^{p}\right)^{2}, \gamma_{i} \geq 0
$$

where $\gamma \in(0,1)$ gives the status of output neuron $i$; similarly, $q_{j}^{p}$ is a real differential function, where $q_{j}^{p}$ is the estimated output value. As per Equation (9), to find the local minima and reduce the error value of the error cost function, the relationship between 
neurons $y$ and $z$ is used, where weights $w^{+}(y, z)$ and $w^{-}(y, z)$ are updated by

$$
w_{y, z}^{+t}=w_{y, z}^{+(t-1)}-\eta \sum_{i=1}^{n} \gamma_{i}\left(q_{j}^{p}-y_{j}^{p}\right)\left[\frac{\partial q_{i}}{\partial w_{y, z}^{+}}\right]^{t-1},
$$

Moreover,

$$
w_{y, z}^{-t}=w_{y, z}^{-(t-1)}-\eta \sum_{i=1}^{n} \gamma_{i}\left(q_{j}^{p}-y_{j}^{p}\right)\left[\frac{\partial q_{i}}{\partial w_{y, z}^{-}}\right]^{t-1} .
$$

More details about RNN and GD are reported in [37]. In this work, RNN is used to develop our proposed model to accurately map the input to the output using the LoRaWAN Antwerp dataset (RSSI values, Latitude, and Longitude coordinates), and the developed model is used to output any desired unknown $X, Y$ position coordinates. This work considers deg2utm stand-alone function application to convert GPS coordinates to $X, Y$ vector coordinates using MATLAB R2020b [48]. RNN supervised learning algorithm is considered for training the proposed model to locate each end device in the network service area, and then the trained model is extended further to predict the position of any other LoRa sensor nodes on the same network grid based on end-device RSSI values, as shown in Figure 4.

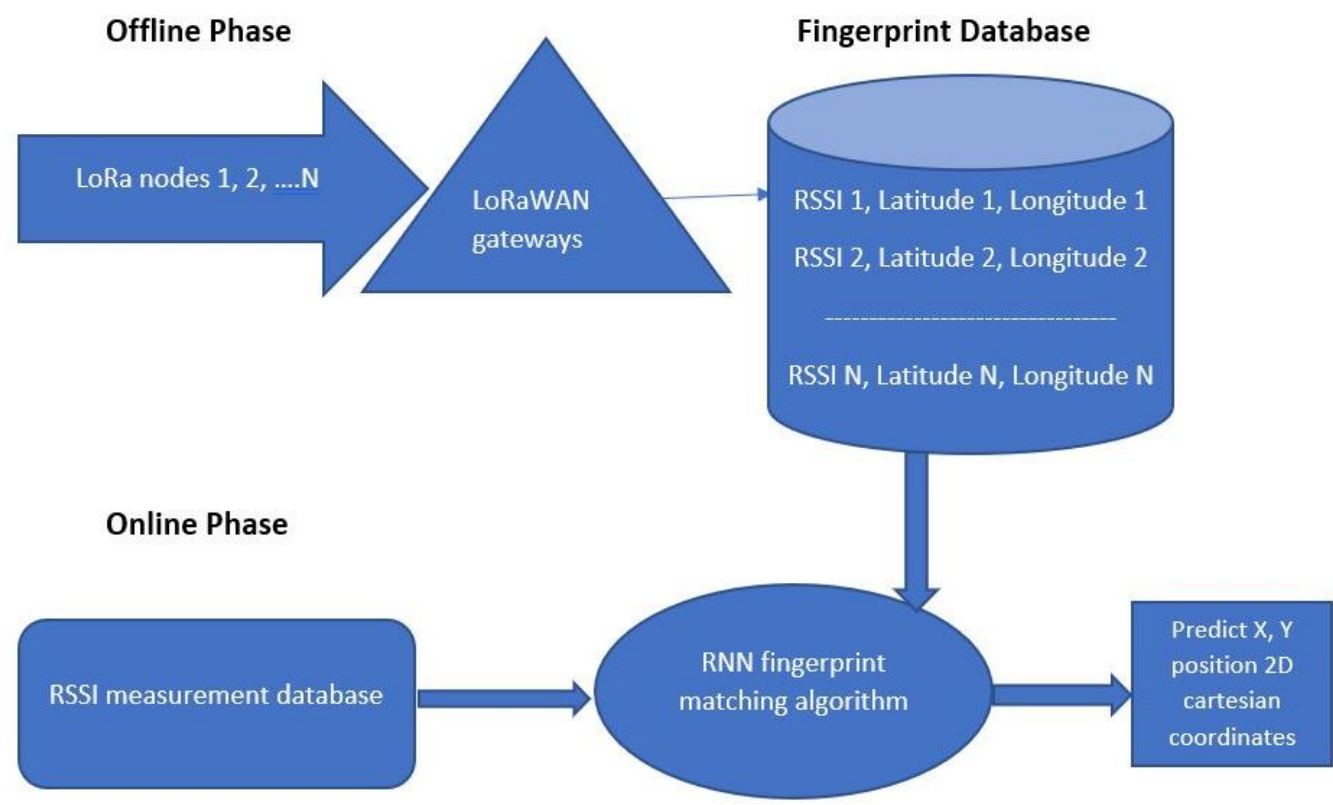

Figure 4. RNN-based RSSI fingerprint localization method.

We used the LoRaWAN Antwerp dataset [46] to train and test our proposed RNNLoRa-based localization using the gradient descent algorithm for regression. Different experiment setups are designed, whereby in the first instance, $80 \%$ of the available 130,343 total data points are used for training the model with the used LR in different epochs, and the remaining $20 \%$ of the dataset is used for testing the model. The proposed RNN model runs k-folds and uses seventy-two input layer neurons, seventy-two hidden layer neurons, and two output layer neurons. Algorithm 1 is the RNN-based localization algorithm used: 


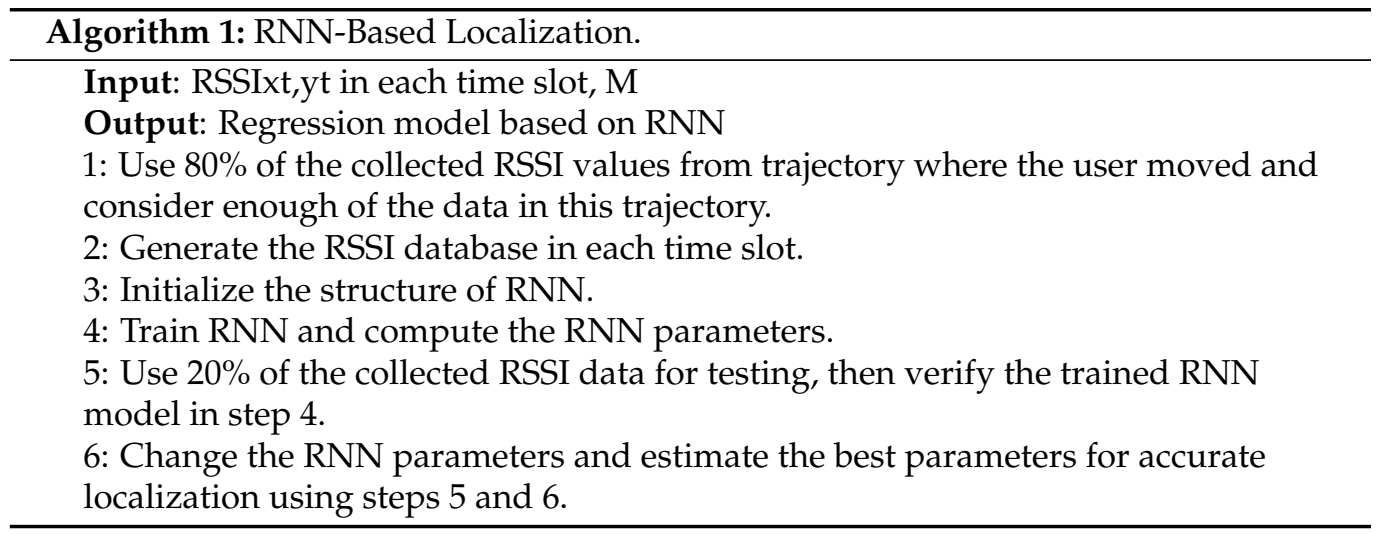

\section{Results and Analysis}

To analyze the performance and localization accuracy of our proposed RNN model, we used the MATLAB R2020b simulation-controlled environment. Figure 5 shows the system's average mean localization error values with the used learning rates of $0.001,0.01$, 0.1 , and 1 at different epochs. The developed RNN-based localization model was evaluated using the average localization error $(\mathrm{AE})$ defined by the following formula:

$$
A E=\sum_{i=1}^{n}\left(\left(X_{\text {real }}-X_{\text {pred }}\right)^{2}+\left(Y_{\text {real }}-Y_{\text {pred }}\right)^{2}\right)^{0.5}
$$

where $\left(X_{\text {real }}, Y_{\text {real }}\right)$ is the actual prerecorded position coordinates recorded using GPS; $\left(X_{\text {pred }}, Y_{\text {pred }}\right)$ is the estimated location of unknown location predicted by the developed localization system; and $\mathrm{n}$ represents the total number of data samples used in modeling.

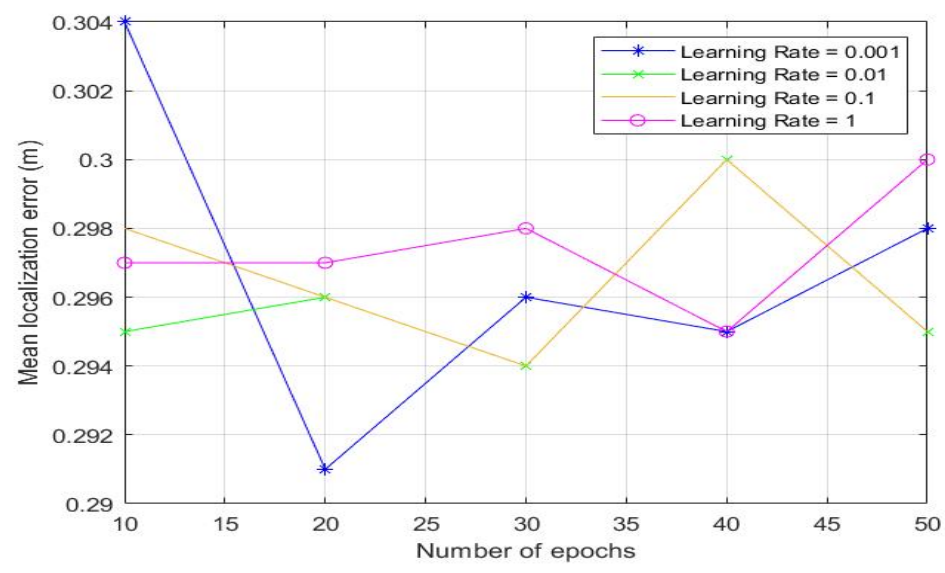

Figure 5. Mean localization error for the used learning rates with various numbers of epochs.

From Figure 6, generally, the highest accuracy of the proposed model was obtained with lower learning rates than higher learning rates at the expense of longer training time. Additionally, with higher learning rates, the localization system tends to be more unstable during training. We trained our model with multiple learning rates to offset these issues. Increasing the learning rate from 0.001 to 0.1 did not improve the developed system's accuracy by increasing the system's mean localization error by $0.03 \mathrm{~m}$, which may be significant or not depending on the application. Meanwhile, increasing the learning rate further to 1 improved the accuracy of the localization system by minimizing the mean localization error by $0.02 \mathrm{~m}$. Our system achieved a minimum mean localization error of $0.291 \mathrm{~m}$ while using a learning rate of 0.001 . More details about obtained mean localization error values obtained for all the used learning rates are presented in Table 1. Additionally, a minimum mean square error (MSE) value of $0.09 \mathrm{~m}$ was obtained while training our model. 


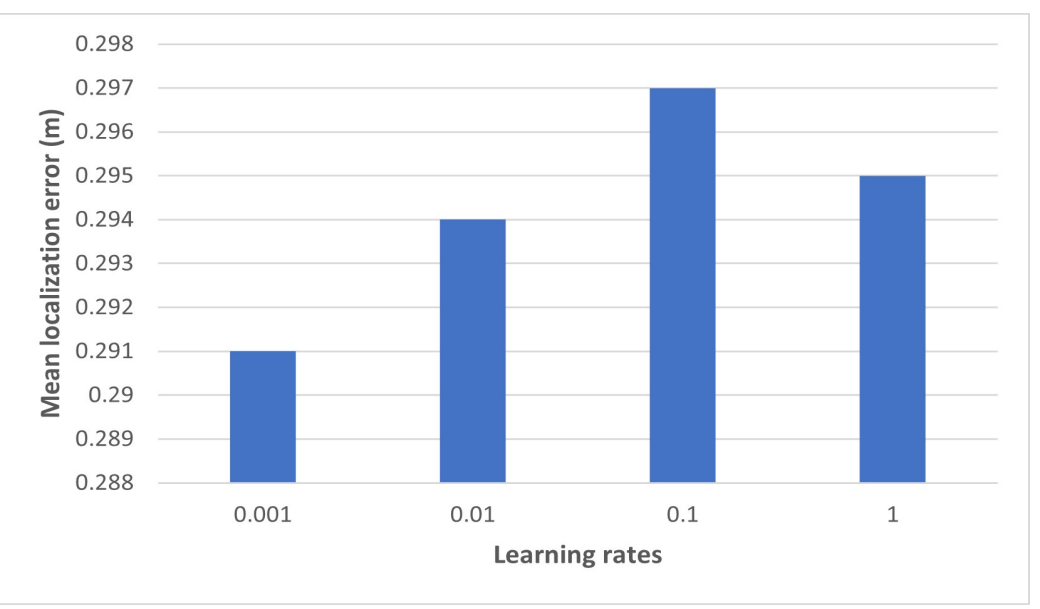

Figure 6. Minimum mean localization error values for the used learning rates.

Table 1. Average mean localization error $(\mathrm{m})$ for used learning rates.

\begin{tabular}{cc}
\hline Learning Rate & Mean Localization Error $(\mathbf{m})$ \\
\hline 0.001 & 0.291 \\
0.01 & 0.294 \\
0.1 & 0.297 \\
1 & 0.295 \\
\hline
\end{tabular}

Next, we evaluated the impact of the different samples since we had a massive number of data points that took a very long training time, depending on the used learning rate. The mean localization error values of our system while using 1000, 3000, 5000, 10,000, and 15,000 data samples keeping the same RNN network architecture with the same used learning rates plus 0.0001 are shown in Figure 7. For 1000, 5000, and 10,000 data samples, increasing the learning rate from 0.0001 to 0.01 increased the mean localization error of the system, and when we increased further to 0.1 and 1, the system's error decreased. Furthermore, for 3000 and 15,000 data samples, increasing the learning rate from 0.0001 to 1 improved the system's performance by decreasing its mean localization error.

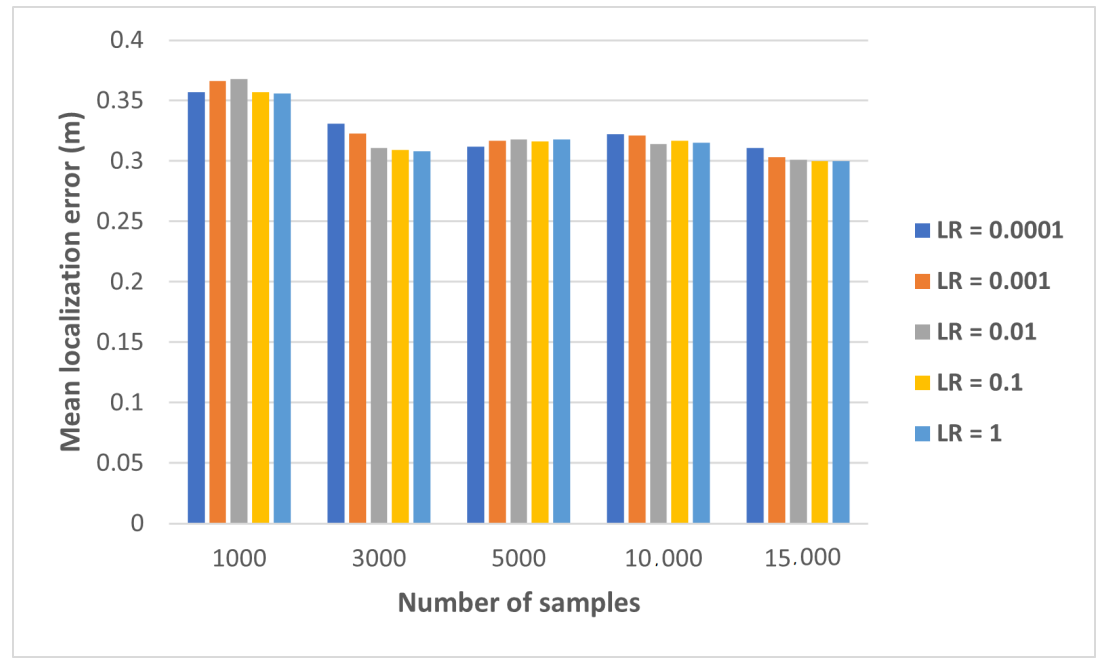

Figure 7. Mean localization error for the used samples.

The minimum mean localization error of $0.3 \mathrm{~m}$ was achieved with 15,000 samples while using learning rates of 0.1 and 1 . Consequently, we investigated the total RNN training time elapsed (in sec) for the used number of samples and learning rates, as shown in Table 2. Hence, the highest system accuracy was achieved using the highest learning rate 
of one with the shortest training time of $4970 \mathrm{~s}$ and a training error of MSE equal to $0.1 \mathrm{~m}$. Mean localization values obtained for all the used samples are presented in Figure 8 with more details given in Table 2. The obtained results about data samples show that increasing data samples from 1000 to 15,000 and further, to 130,343 samples, did not significantly impact the system's accuracy as the difference is only $0.01 \mathrm{~m}$ at the expense of higher training times.

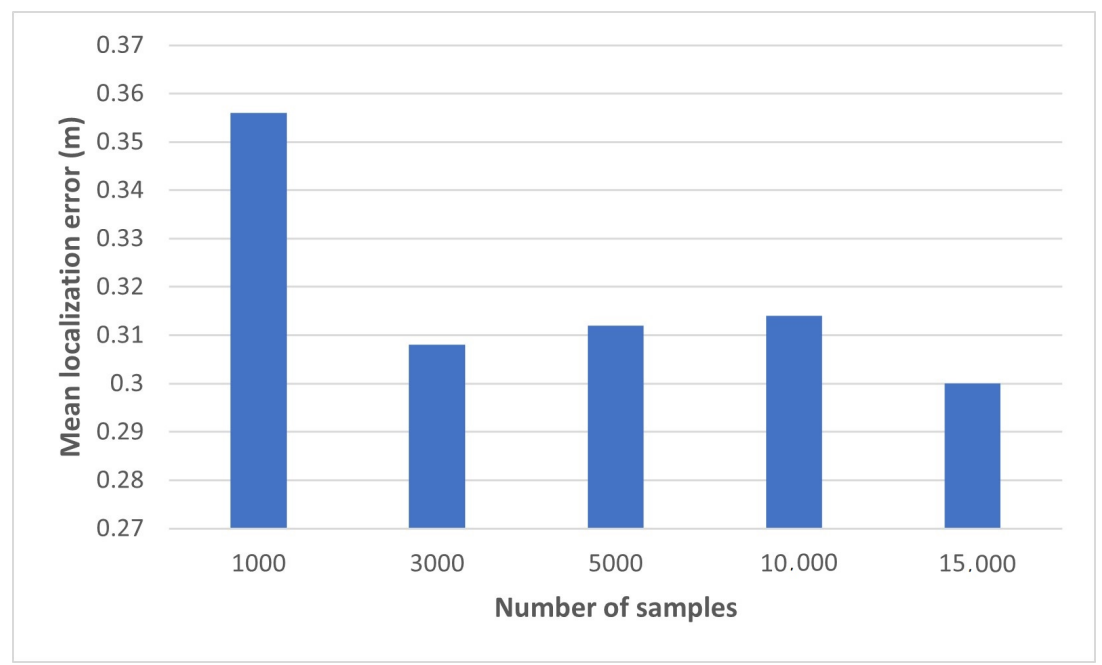

Figure 8. Minimum mean localization error values for the used samples.

Table 2. Average mean localization error (m) for used samples.

\begin{tabular}{cc}
\hline Samples & Mean Localization Error $(\mathbf{m})$ \\
\hline 1000 & 0.356 \\
3000 & 0.308 \\
5000 & 0.312 \\
10,000 & 0.314 \\
15,000 & 0.3 \\
\hline
\end{tabular}

\section{Comparative Performance Analysis}

The localization accuracy of the proposed RNN-based localization approach is compared with traditional localization approaches presented in related research studies, and Table 3 summarizes the localization performance of each of the systems. A minimum mean localization error of $0.39 \mathrm{~m}$ was achieved for a small-scale urban area in our previously published work [45]. Bonafini et al. [49] used Multilateration algorithm and achieved a minimum localization error of $6.2 \mathrm{~m}$ while Du et al. [50] obtained a minimum localization error of $7.57 \mathrm{~m}$. Shokry et al. [51] used deep learning and obtained a minimum localization error of $18.8 \mathrm{~m}$, and Anjum et al. [52] used a linear regression model and achieved a localization error of $45.75 \mathrm{~m}$. Purohit et al. [53] used deep neural networks and obtained a localization error of $191.52 \mathrm{~m}$; Janssen et al. [54] also used kNN and obtained a localization error of $340 \mathrm{~m}$. Aernouts et al. [46] used kNN method and achieved a localization error of $398.4 \mathrm{~m}$; Anagnostopoulos et al. [8] also used ANN and obtained an error of $358 \mathrm{~m}$, while Nguyen [55] used ANN approach and achieved a localization error of $500 \mathrm{~m}$. From Table 3, it is clear that the proposed RNN-based localization model outperforms the other RSSI fingerprint LoRaWAN-based localization systems from related research with a minimum localization error of $0.29 \mathrm{~m}$. The proposed RNN method has achieved $25 \%, 95 \%, 96 \%, 98.5 \%, 99.85 \%, 99.37 \%, 99.92 \%, 99.93 \%$, and $99.94 \%$ improvements in mean localization error compared with our previous work [45], Bonafini et al. [49], Du et al. [50], Shokry et al. [51], Anjum et al. [52], Purohit et al. [53], Janssen et al. [54], Aernouts et al. [46], Anagnostopoulos et al. [8], and Nguyen [55], respectively. 
Table 3. Accuracy of different RSSI fingerprinting approaches in outdoor LoRaWAN from different research studies.

\begin{tabular}{ccc}
\hline Research Work & Mean Localization Error (m) & Approach \\
\hline Proposed RNN-based localization system & 0.29 & RNN \\
Ingabire et al. [45] & 0.39 & RNN \\
Bonafini et al. [49] & 6.2 & Multilateration \\
Du et al. [50] & 7.57 & Hybrid \\
Shokry et al. [51] & 18.8 & Deep learning \\
Anjum et al. [52] & 45.75 & Linear \\
Purohit et al. [53] & 191.52 & ANN-Deep \\
Janssen et al. [54] & 340 & kNN \\
Aernouts et al. [46] & 398.4 & kNN \\
Anagnostopoulos et al. [8] & 358 & ANN \\
Nguyen [55] & 500 & ANN \\
\hline
\end{tabular}

\section{Conclusions and Future Work}

In this paper, we proposed a LoRa RSSI-based localization system using the RNN algorithm. The proposed method uses 0.001, 0.01, 0.1 learning rates and 1000, 3000, 5000 , and 10,000 data samples to evaluate the performance of the proposed RNN-LoRa RSSI-based localization system in a large, dense urban area of Antwerp in Belgium. The proposed localization system is trained using $0.001,0.01,0.1$, and 1 learning rates; 1000, $3000,5000,10,000$, and 130,343 data samples; and about 1000 data samples were enough to evaluate our system's performance with minimum training time. Furthermore, through extensive simulations and analysis, the proposed system tested and achieved a minimum mean localization error of $0.29 \mathrm{~m}$. We plan to evaluate the performance of our proposed localization system in an indoor environment.

Author Contributions: Writing—original draft, W.I.; writing—review and editing, H.L., R.M.G. and A.-U.-H.Q. All authors have read and agreed to the published version of the manuscript.

Funding: This work was supported by the Commonwealth Scholarships in the UK in partnership with the Government of Rwanda.

Informed Consent Statement: Not applicable.

Data Availability Statement: Not applicable.

Conflicts of Interest: The authors declare no conflict of interest.

\section{References}

1. Poulose, A.; Kim, J.; Han, D.S. A sensor fusion framework for indoor localization using smartphone sensors and Wi-Fi RSSI measurements. Appl. Sci. 2019, 9, 4379. [CrossRef]

2. Yang, Z.; Schafer, J.; Ganz, A. Disaster response: Victims' localization using Bluetooth Low Energy sensors. In Proceedings of the 2017 IIEEE International Symposium on Technologies for Homeland Security (HST), Waltham, MA, USA, 25-26 April 2017; pp. 31-34.

3. Uradzinski, M.; Guo, H.; Liu, X.; Yu, M. Advanced Indoor Positioning Using Zigbee Wireless Technology. Wirel. Pers. Commun. 2017, 97, 6509-6518. [CrossRef]

4. Richter, P.; Toledano-Ayala, M. Ubiquitous and Seamless Localization: Fusing GNSS Pseudoranges and WLAN Signal Strengths. Mob. Inf. Syst. 2017, 2017, 8260746. [CrossRef]

5. Kang, J.M.; Yoon, T.S.; Kim, E.; Park, J.B. Lane-level map-matching method for vehicle localization using GPS and camera on a high-definition map. Sensors 2020, 20, 2166. [CrossRef] [PubMed]

6. Daramouskas, I.; Kapoulas, V.; Pegiazis, T. A survey of methods for location estimation on Low Power Wide Area Networks. In Proceedings of the 2019 10th International Conference on Information, Intelligence, Systems and Applications (IISA), Patras, Greece, 15-17 July 2019; pp. 2019-2022.

7. Podevijn, N.; Trogh, J.; Karaagac, A.; Haxhibeqiri, J.; Hoebeke, J.; Martens, L.; Suanet, P.; Hendrikse, K.; Plets, D.; Joseph, W. TDoA-based outdoor positioning in a public LoRa network. In Proceedings of the 12th European Conference on Antennas and Propagation (EuCAP 2018), London, UK, 9-13 April 2018. 
8. Anagnostopoulos, G.G.; Kalousis, A. A Reproducible Comparison of RSSI Fingerprinting Localization Methods Using LoRaWAN. In Proceedings of the 2019 16th Workshop on Positioning, Navigation and Communications (WPNC), Bremen, Germany, 23-24 October 2019.

9. Podevijn, N.; Plets, D.; Trogh, J.; Martens, L.; Suanet, P.; Hendrikse, K.; Joseph, W. TDoA-Based Outdoor Positioning with Tracking Algorithm in a Public LoRa Network. Wirel. Commun. Mob. Comput. 2018, 2018, 1864209. [CrossRef]

10. Choi, W.; Chang, Y.S.; Jung, Y.; Song, J. Low-power LORa signal-based outdoor positioning using fingerprint algorithm. ISPRS Int.-Geo-Inf. 2018, 7, 440. [CrossRef]

11. Bhatti, G. Machine learning based localization in large-scale wireless sensor networks. Sensors 2018, 18, 4179. [CrossRef]

12. Qureshi, A.U.H.; Larijani, H.; Ahmad, J.; Mtetwa, N. A Novel Random Neural Network Based Approach for Intrusion Detection Systems. In Proceedings of the 2018 10th Computer Science and Electronic Engineering (CEEC), Colchester, UK, 19-21 September 2018; pp. 50-55.

13. Semtech. LoRaWANspecificationv1.1. Available online: https://www.lora-alliance.org/technology (accessed on 30 August 2021).

14. Bissett, D. Analysing Tdoa Localisation in LoRa Networks; Delft University of Technology: Delft, The Netherlands, 2018.

15. Ha, G.Y.; Seo, S.B.; Oh, H.S.; Jeon, W.S. LoRa ToA-based localization using fingerprint method. In Proceedings of the 2019 International Conference on Information and Communication Technology Convergence (ICTC), Jeju, Korea, 16-18 October 2019; pp. 349-353.

16. Fargas, B.C.; Petersen, M.N. GPS-free geolocation using LoRa in low-power WANs. In Proceedings of the 2017 Global Internet of Things Summit (Giots), Geneva, Switzerland, 6-9 June 2017.

17. Walravens, M.; Verreyken, E.; Steckel, J. Spiking neural network implementation on fpga for robotic behaviour. In Proceedings of the International Conference on P2P, Parallel, Grid, Cloud and Internet Computing 2019, Antwerp, Belgium, 7-9 November 2019; pp. 694-703.

18. Abdul, N.; Zghair, K.; Croock, M.S.; Abdul, A.; Taresh, R. Indoor Localization System Using Wi-Fi Technology. Iraqi J. Comput. Commun. Control Syst. Eng. 2019, 19, 69-77.

19. Hernández, N.; Ocaña, M.; Alonso, J.M.; Kim, E. Continuous space estimation: Increasingwifi-based indoor localization resolution without increasing the site-survey effort. Sensors 2017, 17, 147. [CrossRef] [PubMed]

20. Janssen, T.; Weyn, M.; Berkvens, R. Localization in low power wide area networks using wi-fi fingerprints. Appl. Sci. 2017, 7, 936. [CrossRef]

21. Zafari, F.; Gkelias, A.; Leung, K.K. A Survey of Indoor Localization Systems and Technologies. IEEE Commun. Surv. Tutor. 2019, 21, 2568-2599. [CrossRef]

22. Sadowski, S.; Spachos, P. RSSI-Based Indoor Localization with the Internet of Things. IEEE Access 2018, 6, 30149-30161. [CrossRef]

23. Lin, Y.; Dong, W.; Gao, Y.; Gu, T. SateLoc: A virtual fingerprinting approach to outdoor lora localization using satellite images. In Proceedings of the 19th ACM/IEEE International Conference on Information Processing in Sensor Networks (IPSN 2020), Sydney, Australia, 21-24 April 2020; pp. 13-24. [CrossRef]

24. Samanta, R.; Kumari, C.; Deb, N.; Bose, S.; Cortesi, A.; Chaki, N. Node localization for indoor tracking using artificial neural network. In Proceedings of the 2018 Third International Conference on Fog and Mobile Edge Computing (FMEC), Barcelona, Spain, 23-26 April 2018; pp. 229-233.

25. Bhatnagar, T. Application of Artificial Intelligence and Low- Power Wide-Area Network (LPWAN) in the development of Smart Cities. In Unmanned Aerial Vehicles in Smart Cities; Springer International Publishing: New York, NY, USA, 2020.

26. Gu, C.; Tan, R. LoRa-Based Localization: Opportunities and Challenges LoRa-Based Localization: Opportunities and Challenges. arXiv 2018, arXiv:1812.11481.

27. Daramouskas, I.; Kapoulas, V.; Paraskevas, M. Using Neural Networks for RSSI Location Estimation in LoRa Networks. In Proceedings of the 2019 10th International Conference on Information, Intelligence, Systems and Applications (IISA), Patras, Greece, 15-17 July 2019; pp. 1-7.

28. Sallouha, H.; Chiumento, A.; Rajendran, S.; Pollin, S. Localization in Ultra Narrow Band IoT Networks: Design Guidelines and Tradeoffs. IEEE Internet Things J. 2019, 6, 9375-9385. [CrossRef]

29. Adege, A.B.; Lin, H.P.; Tarekegn, G.B.; Jeng, S.S. Applying deep neural network (DNN) for robust indoor localization in multi-building environment. Appl. Sci. 2018, 8, 1062. [CrossRef]

30. Xu, B.; Zhu, X.; Zhu, H. An efficient indoor localization method based on the long short-term memory recurrent neuron network. IEEE Access 2019, 7, 123912-123921. [CrossRef]

31. Wu, L.; Chen, C.H.; Zhang, Q. A mobile positioning method based on deep learning techniques. Electronics 2019, 8, 59. [CrossRef]

32. Hoang, M.T.; Yuen, B.; Dong, X.; Lu, T.; Westendorp, R.; Reddy, K. Recurrent Neural Networks for Accurate RSSI Indoor Localization. IEEE Internet Things J. 2019, 6, 10639-10651. [CrossRef]

33. Carrino, F.; Janka, A.; Khaled, O.A.; Mugellini, E. LoRaLoc: Machine Learning-Based Fingerprinting for Outdoor Geolocation using LoRa. In Proceedings of the 2019 6th Swiss Conference on Data Science (SDS), Bern, Switzerland, 14 June 2019 ; pp. 82-86.

34. Javed, A.; Larijani, H.; Ahmadinia, A.; Emmanuel, R.; Mannion, M.; Gibson, D. Design and Implementation of a Cloud Enabled Random Neural Network-Based Decentralized Smart Controller with Intelligent Sensor Nodes for HVAC. IEEE Internet Things J. 2017, 4, 393-403. [CrossRef] 
35. Javed, A.; Larijani, H.; Wixted, A.; Emmanuel, R. Random neural networks based cognitive controller for HVAC in non-domestic building using LoRa. In Proceedings of the 2017 IEEE 16th International Conference on Cognitive Informatics \& Cognitive Computing (ICCI* CC), Oxford, UK, 26-28 July 2017; pp. 220-226.

36. Javed, A.; Larijani, H.; Ahmadinia, A.; Gibson, D. Smart Random Neural Network Controller for HVAC Using Cloud Computing Technology. IEEE Trans. Ind. Inform. 2017, 13, 351-360. [CrossRef]

37. Ahmad, J.; Larijani, H.; Emmanuel, R.; Mannion, M.; Javed, A.; Phillipson, M. Energy demand prediction through novel random neural network predictor for large non-domestic buildings. In Proceedings of the 2017 Annual IEEE International Systems Conference (SysCon), Montreal, QC, Canada, 24-27 April 2017; pp. 1-6.

38. Simonyan, K.; Zisserman, A. Very deep convolutional networks for large-scale image recognition. In Proceedings of the 3rd International Conference on Learning Representations (ICLR 2015), San Diego, CA, USA, 7-9 May 2015; pp. 1-14.

39. Qureshi, A.U.H.; Larijani, H.; Javed, A.; Mtetwa, N.; Ahmad, J. Intrusion Detection Using Swarm Intelligence. In Proceedings of the 2019 UK/China Emerging Technologies (UCET), Glasgow, UK, 21-22 August 2019; pp. 1-5.

40. Qureshi, A.U.H.; Larijani, H.; Mtetwa, N.; Javed, A.; Ahmad, J. RNN-ABC: A new swarm optimization based technique for anomaly detection. Computers 2019, 8, 59. [CrossRef]

41. Cerkez, C.; Aybay, I.; Halici, U. A digital neuron realization for the random neural network model. In Proceedings of the International Conference on Neural Networks (ICNN'97), Houston, TX, USA, 12 June 1997; Volume 2, pp. $1000-1004$.

42. Abdelbaki, H.; Gelenbe, E.; El-Khamy, S.E. Analog hardware implementation of the random neural network model. In Proceedings of the IEEE-INNS-ENNS International Joint Conference on Neural Networks, Como, Italy, 27 July 2000; Volume 4, pp. 197-201.

43. Abdelbaki, H. Random Neural Network Simulator (Rnnsim) v. 2. Free Simulator. 1999. Available online: ftp:/ ftp.mathworks. $\mathrm{com} / \mathrm{pub} / \mathrm{contrib} / \mathrm{v} 5 /$ nnet/rnnsimv2 (accessed on 30 August 2021).

44. Mohamed, S.; Rubino, G. A study of real-time packet video quality using random neural networks. IEEE Trans. Circuits Syst. Video Technol. 2002, 12, 1071-1083. [CrossRef]

45. Ingabire, W.; Larijani, H.; Gibson, R.M. LoRa RSSI Based Outdoor Localization in an Urban Area Using Random Neural Networks. In Intelligent Computing; Lecture Notes in Networks and Systems; Arai, K., Ed.; Springer: Cham, Switzerland, 2021 ; Volume 284. [CrossRef]

46. Aernouts, M.; Berkvens, R.; Vlaenderen, K.V.; Weyn, M. Sigfox and LoRaWAN datasets for fingerprint localization in large urban and rural areas. Data 2018, 3, 13. [CrossRef]

47. Gelenbe, E. Random Neural Networks with Negative and Positive Signal and Product Form Solution. Neural Comput. 1989, 1, 502-510 [CrossRef]

48. Rafael Palacios. Deg2utm. MATLAB Central File Exchange. Available online: https://www.mathworks.com/matlabcentral/ fileexchange/10915-deg2utm (accessed on 30 August 2021).

49. Bonafini, F.; Carvalho, D.F.; Depari, A.; Ferrari, P.; Flammini, A.; Pasetti, M.; Rinaldi, S.; Sisinni, E. Evaluating indoor and outdoor localization services for LoRaWAN in Smart City applications. In Proceedings of the 2019 II Workshop on Metrology for Industry 4.0 and IoT (MetroInd4. 0\&IoT), Naples, Italy, 4-6 June 2019; pp. 300-305.

50. Du, H.; Zhang, C.; Ye, Q.; Xu, W.; Kibenge, P.L.; Yao, K. A hybrid outdoor localization scheme with high-position accuracy and low-power consumption. Eurasip J. Wirel. Commun. Netw. 2018, 2018, 4. [CrossRef]

51. Shokry, A.; Torki, M.; Youssef, M. DeepLoc: A Ubiquitous Accurate and Low-Overhead Outdoor Cellular Localization System. In Proceedings of the 26th ACM SIGSPATIAL International Conference on Advances in Geographic Information Systems, Seattle, WA, USA, 6-9 November 2021; pp. 27-29.

52. Anjum, M.; Khan, M.A.; Hassan, S.A.; Mahmood, A.; Qureshi, H.K. RSSI Fingerprinting-based Localization Using Machine Learning in LoRa Networks. IEEE Internet Things Mag. 2020, 3, 53-59. [CrossRef]

53. Purohit, J.N.; Wang, X. LoRa Based Localization Using Deep Learning Techniques; California State University: Long Beach, CA, USA, 2019.

54. Janssen, T.; Aernouts, M.; Berkvens, R.; Weyn, M. Outdoor Fingerprinting Localization using Sigfox. In Proceedings of the 2018 International Conference on Indoor Positioning and Indoor Navigation (IPIN), Nantes, France, 24-27 September 2018. [CrossRef]

55. Nguyen, T.A. LoRa Localisation in Cities with Neural Networks; Delft University of Technology: Delft, The Netherlands, 2019 ; p. 71. 